\title{
Bur open Delirium in an adult acute hospital population: predictors, prevalence and detection
}

\author{
Daniel James Ryan, ${ }^{1}$ Niamh Annmarie O’Regan, ${ }^{1}$ Ronán Ó Caoimh, ${ }^{1}$ Josie Clare ${ }^{2}$ \\ Marie O'Connor, ${ }^{3}$ Maeve Leonard, ${ }^{4}$ John McFarland, ${ }^{5}$ Sheila Tighe, ${ }^{6}$ \\ Kathleen O'Sullivan, ${ }^{7}$ Paula T Trzepacz, ${ }^{8,9}$ David Meagher, ${ }^{4}$ Suzanne Timmons ${ }^{1}$
}

To cite: Ryan DJ, O'Regan NA, Ó Caoimh R, et al. Delirium in an adult acute hospital population: predictors, prevalence and detection. BMJ Open 2013;3:e001772.

doi:10.1136/bmjopen-2012001772

- Prepublication history for this paper are available online. To view these files please visit the journal online (http://dx.doi.org/10.1136/ bmjopen-2012-001772).

DJR and NAO contributed equally to this study.

Data were presented by DM at the American Delirium Society 2nd annual meeting, Indianapolis, Indiana, USA, June 2012

Received 24 August 2012 Revised 1 November 2012 Accepted 15 November 2012

This final article is available for use under the terms of the Creative Commons Attribution Non-Commercial 2.0 Licence; see http://bmjopen.bmj.com

For numbered affiliations see end of article.

Correspondence to Dr Niamh O'Regan; niamh.oregan@ucc.ie

\section{ABSTRACT}

Background: To date, delirium prevalence and incidence in acute hospitals has been estimated from pooled findings of studies performed in distinct patient populations.

Objective: To determine delirium prevalence across an acute care facility.

Design: A point prevalence study.

Setting: A large tertiary care, teaching hospital.

Patients: 311 general hospital adult inpatients were assessed over a single day. Of those, 280 had full data collected within the study's time frame $(90 \%)$.

Measurements: Initial screening for inattention was performed using the spatial span forwards and months backwards tests by junior medical staff, followed by two independent formal delirium assessments: first the Confusion Assessment Method (CAM) by trained geriatric medicine consultants and registrars, and, subsequently, the Delirium Rating Scale-Revised-98 (DRS-R98) by experienced psychiatrists. The diagnosis of delirium was ultimately made using DSM-IV (Diagnostic and Statistical Manual of Mental Disorders, Fourth Edition) criteria.

Results: Using DSM-IV criteria, 55 of 280 patients $(19.6 \%)$ had delirium versus $17.6 \%$ using the CAM. Using the DRS-R98 total score for independent diagnosis, $20.7 \%$ had full delirium, and $8.6 \%$ had subsyndromal delirium. Prevalence was higher in older patients ( $4.7 \%$ if $<50$ years and $34.8 \%$ if $>80$ years) and particularly in those with prior dementia (OR=15.33, $p<0.001$ ), even when adjusted for potential confounders. Although $50.9 \%$ of delirious patients had pre-existing dementia, it was poorly documented in the medical notes. Delirium symptoms detected by medical notes, nurse interview and patient reports did not overlap much, with inattention noted by professional staff, and acute change and sleep-wake disturbance noted by patients.

Conclusions: Our point prevalence study confirms that delirium occurs in about $1 / 5$ of general hospital inpatients and particularly in those with prior cognitive impairment. Recognition strategies may need to be tailored to the symptoms most noticed by the detector (patient, nurse or primary physician) if formal assessments are not available.

\section{ARTICLE SUMMARY}

Article focus

- Delirium prevalence in the acute hospital has, to date, been estimated from pooled findings from many studies within individual units. The main focus of this study was to ascertain the point prevalence of delirium collectively across almost an entire tertiary referral centre.

- Additionally, we sought to evaluate the rates of delirium detection by medical and nursing staff and to describe which features alerted staff members to the presence of delirium.

\section{Key messages}

- Delirium is a very common problem in the acute hospital setting, with a point prevalence of approximately $20 \%$.

- Advancing age and pre-existing cognitive impairment were independently associated with a higher prevalence of delirium, in keeping with previous studies.

- Medical and nursing staff detect different delirium features, which has clear implications for staff education and training: nursing staff notice inattentiveness, lability of affect and delusions, whereas medical staff tend to detect delirium in the presence of short-term memory impairment in addition to inattention.

\section{INTRODUCTION}

Delirium is a complex neuropsychiatric syndrome, commonly encountered across all healthcare settings, and associated with adverse outcomes including more prolonged hospitalisation, institutionalisation and increased mortality, independent of age, prior cognitive functioning and comorbidities. ${ }^{1} 2$ In a recent study, mortality was shown to increase by $11 \%$ for every additional $48 \mathrm{~h}$ of active delirium, highlighting the need for timely detection and treatment. ${ }^{3}$ However, delirium is misdiagnosed, detected late or missed in over $50 \%$ of cases across healthcare 


\section{ARTICLE SUMMARY}

Strengths and limitations of this study

- This study is the first to collectively assess the point prevalence of delirium across an acute hospital, which was largely consistent using three different diagnostic methods.

- Confusion Assessment Method assessors were vigorously trained in its use, and Delirium Rating Scale-Revised-98 and Diagnostic and Statistical Manual of Mental Disorders, Fourth Edition criteria were applied by highly experienced delirium researchers.

- In order to ensure the feasibility of the study, we adopted a screening process (including tests of attention, a core delirium component), and only those who screened positive went on to have formal delirium assessment. Our high rate of participation indicates that our methods were acceptable to patients and, hence, may be appropriate for routine screening on the ground.

- The true hospital-wide point prevalence of delirium is likely to be slightly higher than our figure of $20.7 \%$, given that those who were excluded from the study would be expected to have higher rates of delirium overall (patients in intensive care unit, Burns unit, stroke patients with aphasia).

settings. ${ }^{4}$ This under-recognition, along with increasing evidence regarding delirium treatment, ${ }^{5}$ emphasises the importance of understanding the factors that relate to accurate identification.

A meta-analysis of 42 studies reported delirium to be prevalent on admission in 10-31\% of medical inpatients, and to occur in the hospital in $3-29 \% .^{6}$ However, these estimates are based on pooled findings from multiple studies within individual services or units, rather than a more complete study of a single hospital, and are limited by terminology differences and diagnostic tool heterogeneity. Thus, a definitive and accurate determination of inhospital delirium prevalence, using standardised delirium instruments, is needed. We report the first hospital-wide point prevalence study of delirium in a large acute hospital within a $24 \mathrm{~h}$ period using three methods of detection. We also compared the delirium prevalence across ward types, between specific patient groups, describing the 'footprint' of delirium. Finally, we explored the delirium features that alerted the attending medical and nursing staff to the presence of delirium as compared to what the patients noticed.

\section{METHODS}

The study was conducted at Cork University Hospital, a tertiary referral, level-one trauma centre in Southern Ireland with 407 acute adult inpatient beds (excluding psychiatry), serving a population of 500000 people. All adult inpatients on 15 May 2010 were to be eligible for inclusion, excluding patients in the emergency department (ED), intensive care units (ICU) and isolation rooms on the Haematology/Burns Isolation Unit, leaving a possible eligible population of 358 patients. To be able to detect with $95 \%$ power a $10 \%$ difference in prevalence in our hospital from the previously reported prevalence in hospitalised patients (10-31\%, Siddiqi et $a t^{6}$ ), a sample size of 267 would be required. Thus, it seemed feasible to recruit sufficient patients to perform this study. On the day of the study, patients were excluded if they were severely aphasic, comatose, dying or deemed too unwell for interview by nursing staff; or refused to participate. All included patients were screened for delirium within a $6 \mathrm{~h}$ period in three phases of assessment, as follows.

\section{Phase 1}

\section{Formal cognitive screening}

Trained junior medical staff screened all patients for inattention using (1) Spatial Span Forwards (SSF), a visual pattern-recognition test that assesses visuospatial memory and attention ${ }^{7}$ and (2) Months Backwards (MB), adapted from the Short Blessed Test for dementia. ${ }^{9}$ Significant inattention was present in those who scored $<5$ on the SSF or were unable to correctly recite $\geq 5$ months backwards starting from December (ie, reaching July without error). In patients with poor visual acuity, counting backwards from 20 to 1 was substituted for the $\mathrm{SSF}^{10}{ }^{10}$ where any error was considered evidence of inattention. Inattention is a cardinal and required symptom for delirium diagnosis and use of a visuospatial attention test has been advocated and shown to highly correlate with delirium. ${ }^{11}$

\section{Patient and healthcare staff recognition of confusion}

Junior staff questioned the patient as to whether they felt currently or recently confused ('have you felt muddled in your thinking, or confused, since you came into hospital?'). Nursing staff were interviewed using standardised questions (see box 1). Medical notes were reviewed for reports of delirium, or the presence of two proxy terms, 'confusion' or 'agitation'.

Box 1 Standard interview questions for researchers with nursing staff to elicit delirium

I have just interviewed__ and I wonder if you could help me form an opinion as to whether he/she has been experiencing delirium or confusion.

1. How many days approximately have you been looking after ?

2. Do you think he/she is delirious or acutely confused?

3. Has there been a sudden change in _'s mental state since coming into hospital?

a. If so, when did you notice this change?

4. Do you think he/she is able to focus well when you are talking to him/her or does he/she tend to ramble off the point?

5. Does he/she seem better at any period in the day compared to other times?

6. Has _ 's level of consciousness been altered at all-has he/ she been drowsy or not interacting, or perhaps hyperaware at times? 
Phase 2

\section{Confusion assessment method}

Any patient with inattention on either test, or who had subjective, nurse-identified or case-note documented confusion, was then formally tested for delirium using the sensitive, short form of the Confusion Assessment Method (CAM) by geriatric medicine consultants and senior trainees on the research team. All had undergone specific training sessions based on the original training manual, ${ }^{12}$ including online case scenario self-assessment sessions (total training time $=8 \mathrm{~h}$ ).

\section{Phase 3}

Delirium Rating Scale-Revised-98 and DSM-IV assessment

Although the CAM is the most commonly used deliriumdetection tool, ${ }^{13}$ recent validation studies suggest that the sensitivity varies considerably between populations and between assessors. ${ }^{14-16}$ Thus, all patients who had CAM assessment were also independently assessed by one of four psychiatrists on the research team with specific expertise in delirium detection, using the Delirium Rating Scale-Revised-98 (DRS-R98). This is a wellvalidated, ${ }^{17-19}$ diagnostically precise tool that rates symptoms over the previous $24 \mathrm{~h}$. It has high inter-rater reliability, validity, sensitivity and specificity for distinguishing delirium from mixed neuropsychiatric conditions including dementia and depression. ${ }^{18-20}$ It is a 16-item scale with 13 severity items (rated 0-3) and three diagnostic items (rated 0-2 or 0-3); total score possible is $0-46$. For this general hospital population, in consultation with the DRS-R98 developer (PTT), and based on the literature, a total score of $0-6$ was taken as normal; 7-11 represented subsyndromal delirium (SSD); and $\geq 12$ points represented full syndromal delirium (FSD).

The presence of delirium was ultimately determined by consensus agreement among the psychiatry panel using gold standard DSM-IV (Diagnostic and Statistical Manual of Mental Disorders, Fourth Edition) criteria. $^{19} 20$ This was based on all available data (attention tests, CAM items, DRS-R98 data, collateral, medical and nursing notes), including the data collected during directly observed DRS-R98 assessment by senior psychiatrists. However, for each individual case, the psychiatrist who performed the DRS-R98 assessment was not involved in the consensus discussion for that particular patient.

\section{Ethical procedures}

It was presumed that many patients would not have capacity to give informed written consent, and so, owing to the non-invasive nature of the study, ethics committee approval was given to augment patient assent with proxy consent from next of kin (where possible) or a responsible caregiver, in accordance with the Helsinki Guidelines for Medical Research involving human subjects. The study objectives and procedures were explained to all patients and those who declined to participate were excluded.
Assessment of previous cognitive status

In all patients with delirium, the medical case notes were reviewed for a diagnosis of preceding cognitive impairment or dementia made by a suitably trained physician. If this was absent, premorbid cognition was determined by telephone interview using the Informant Questionnaire on Cognitive Decline in the Elderly-Short Form (IQCODE-SF), a validated screening tool for detecting cognitive impairment. ${ }^{21} 22$ In patients without delirium less than 65 years of age, if there was no dementia documentation in the case notes, the patient was presumed not to have the condition. As dementia is more common and is known to be under-diagnosed in older people, potentially dementia diagnosis may have been under-reported in the charts. Thus, a random sample of older patients without delirium $(n=40)$ also had baseline preadmission cognition assessed using the IQCODE-SF. Other older people were excluded from analyses involving prior cognitive status.

\section{Other data collected}

Screening investigators also collected data on medication use, blood results (sodium, glucose, thyroid-stimulating hormone, calcium, urea, $\mathrm{C}$ reactive protein, white cell count and albumin level). Current and previous alcohol history was recorded where available in the charts, and the Charlson comorbidity index ${ }^{23}$ was calculated.

\section{Statistical analyses}

All data were analysed using the Statistical Analysis Software program (SAS V.9, SAS Institute, Cary, North Carolina, USA). Pearson $\chi^{2}$ tests and Fisher's exact test examined the relationship between categorical data. Analysis of variance (ANOVA) examined group differences between continuous variables. Unadjusted models were used to examine the effect of various factors on delirium presence (age, gender, medical speciality, baseline cognitive impairment, alcohol excess, Charlson Comorbidity Index, period of ICU stay, number of ward transfers, total medication number, metabolic abnormality, evidence of inflammatory/infective process, hypoalbuminaemia). Variables with $\mathrm{p}<0.25$ were included in a multivariate logistic regression model. The final models were assessed using the Hosmer-Lemeshow $\left(\mathrm{C}_{\mathrm{HL}}\right)$ Test $^{24}$ for goodness of fit, and fitted the data well $(\mathrm{P}>0.05)$. The coefficient of determination (adjusted $\mathrm{R}^{2}$ ) estimated the proportion of variation explained by each model. ${ }^{25}$ Multicollinearity with the independent variables was investigated using condition number, ${ }^{26}$ with no evidence of departures observed. The predictors of delirium recognition by patients, nurses and in case notes were similarly explored by logistic regression analyses using age, gender, pre-existing cognitive state, medical specialty and delirium symptoms measured on the DRS-R98 as possible predictor variables. 


\section{RESULTS}

There were 358 adult inpatients on the study day, excluding the ICU, ED and Haematology/Burns isolation unit. As it was a weekend day, all 5-day wards were closed.

\section{Excluded patients}

Of these 358 inpatients, 5 refused participation; 23 were excluded owing to coma or severe aphasia; 6 were actively dying; 5 had a language barrier; 3 patients were in isolation on a general ward; 3 were undergoing a procedure and 2 were discharged before assessment. Thus, 311 patients were recruited, constituting $86.9 \%$ of the eligible population (figure 1).

Patient demographics are outlined in table1. The median age of included adult inpatients was 69 years (range $17-100$ ) and $51.1 \%$ were female. $57.8 \%$ were over 65 years $(n=180), 24 \%$ were over 80 years $(n=75)$. $51.1 \%$ of patients were under medical care. The median length of hospital stay on the study day was 7 days

\section{Patient demographics}

interquartile range (IQR) 15, and 56.3\% had undergone at least one ward transfer.

\section{Prevalence of delirium}

Of the 311 patients that underwent screening, 162 patients $(52.1 \%)$ either (1) demonstrated inattention using one or more screening tools $(\mathrm{n}=142)$ and/or (2) self-reported confusion $(n=41)$ and/or (3) were identified as confused by nursing staff ( $\mathrm{n}=52)$ and/or (4) had 'confusion' documented in the case notes $(n=29)$. Of these 162 patients, 147 underwent CAM evaluation (90.1\%). CAM omission was due to either patient discharge, transfer to ICU/ isolation, becoming gravely unwell or researcher error $(n=5)$. Of the 296 patients who underwent phase 1 assessment and subsequent CAM, if indicated, $17.6 \% \quad(\mathrm{n}=52)$ were CAM positive (figure 1 ).

Formal evaluation by DRS-R98 and consensus DSM-IV diagnosis was conducted on 133 patients which is somewhat less than the 147 patients who underwent CAM assessment. This was due to similar factors as the CAM omissions. Thus, the complete study protocol was followed in 280 patients. Of these, $19.6 \% \quad(n=55)$ were deemed to have delirium by DSM-IV criteria. Using the

Figure 1 Excluded patients, screened patients and assessment tools used with subsequent consensus opinion regarding DSM-IV status. CAM, Confusion Assessment Method; DRS-R98, Delirium Rating Scale-Revised-98; DSM-IV, Diagnostic and Statistical Manual of Mental Disorders, Fourth Edition.

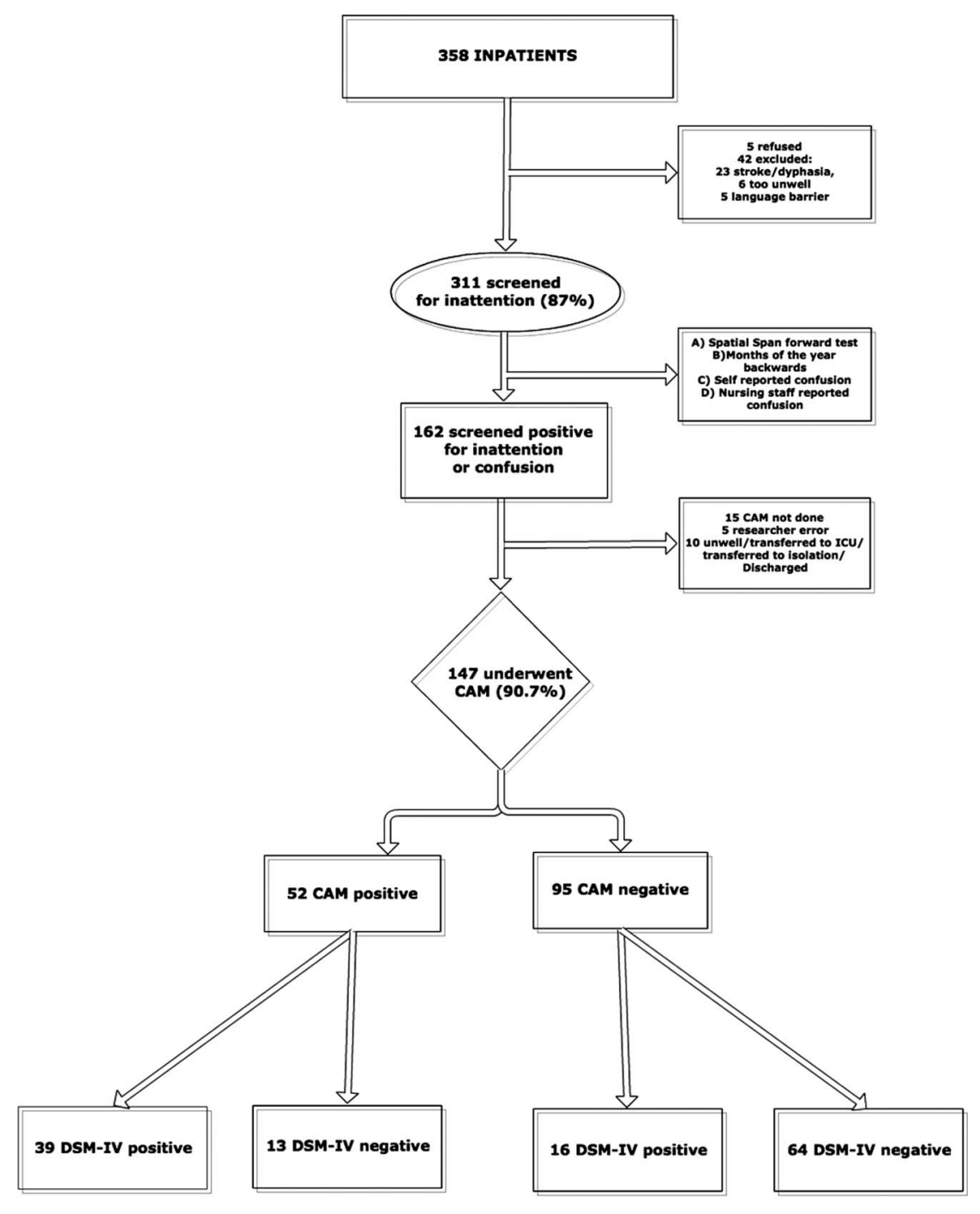


Table 1 Patient's demographical and clinical characteristics by delirium subgroup

\begin{tabular}{lc}
\hline Variable & \\
\hline Age in years, median (range), $\mathrm{n}=311$ & $69(17-100)$ \\
All & $79(34-100)$ \\
Delirium & $66(17-95)$ \\
No delirium & \\
Female sex, $\mathrm{n}(\%), \mathrm{n}=311$ & $159(51.1 \%)$ \\
All & $27(49.1 \%)$ \\
Delirium & $132(51.6 \%)$ \\
No delirium & \\
Length of stay on day of study, median & \\
(IQR), $\mathrm{n}=300$ alive at discharge & $7(15)$ \\
All & $17(33)$ \\
Delirium & $6(14)$ \\
No delirium & \\
Charlson Comorbidity Index, median & \\
(range), $\mathrm{n}=298$ & $2(0-12)$ \\
All & $2(0-12)$ \\
Delirium & $2(0-8)$ \\
No delirium & \\
Dementia, $\mathrm{n}=203$ (\%) & $36(17.7 \%)$ \\
All & \\
Delirium, $\mathrm{n}=55$ & $28(50.9 \%)$ \\
No delirium* $\mathrm{n}=148$ & $8(5.4 \%)$ \\
\hline *May be an underestimate as medical chart documentation used \\
to determine dementia status in younger, non-delirious patients. \\
\end{tabular}

total DRS-R98 score to delineate delirium into FSD (DRS-R98 score $\geq 12$ ) or SSD (score 7-11), 20.7\% $(n=58)$ had FSD, while an additional $8.6 \%(n=24)$ had SSD, with 21 of the SSD patients not meeting DSM-IV criteria for delirium.

Prevalence estimation after adjustment for omitted patients Because not all 311 patients completed the full screening process, we estimated the prevalence of delirium in those who did not undergo delirium testing following a positive screen for inattention/reported confusion $(n=31)$. If these patients were presumed to have a similar prevalence of DSM-IV-diagnosed delirium, we would expect an additional nine delirium cases and a total prevalence of $20.7 \%$ using DSM-IV criteria (figure 2).

\section{Variation in delirium prevalence across the hospital}

A wide variability in delirium prevalence was observed between wards (figure 3, $\mathrm{n}=280$ ). The prevalence was highest on the geriatrics ward $(53.3 \% ; 8 / 15)$, lowest on general surgical wards $(7.2 \% ; 6 / 83)$ and intermediate on general medical wards $(22 \% ; 29 / 131)$ and the orthopaedic ward $(28.6 \% ; 6 / 21)$. Capacity issues within the healthcare service can lead to some patients being admitted to wards that are not designated for their clinical presentation. The prevalence of delirium was therefore also assessed according to the specialty of the designated clinical team. The prevalence of delirium among all medical patients in the hospital was $24.2 \%$ $(39 / 161)$ whereas that of all general surgical specialities was $7.7 \%(6 / 78)$. High-risk surgical patients (orthopaedic and neurosurgical) had prevalence rates equal to medical patients at $24.4 \%(10 / 41)$. The adjusted OR for delirium in a general surgical patient relative to a medical patient was 0.11 (95\% CI 0.03 to $0.41, \mathrm{p}<0.001$, table 2).

\section{Prior cognitive impairment}

Among those diagnosed with delirium using DSM-IV criteria $(n=55), 28(50.9 \%)$ had pre-existing cognitive decline, primarily identified through IQCODE-SF telephone interview (only five had case notes documentation of dementia). An IQCODE-SF was also carried out on 40 age-matched non-delirious inpatients for comparison and nine had cognitive impairment (22.5\%). Using data from all younger patients and only from those

Figure 2 DSM-IV delirium prevalence, including the estimated delirium prevalence in the 31 patients that did not undergo delirium assessment following initial screening. CAM, Confusion Assessment Method; DRS-R98, Delirium Rating Scale-Revised-98; DSM-IV, Diagnostic and Statistical Manual of Mental Disorders, Fourth Edition.

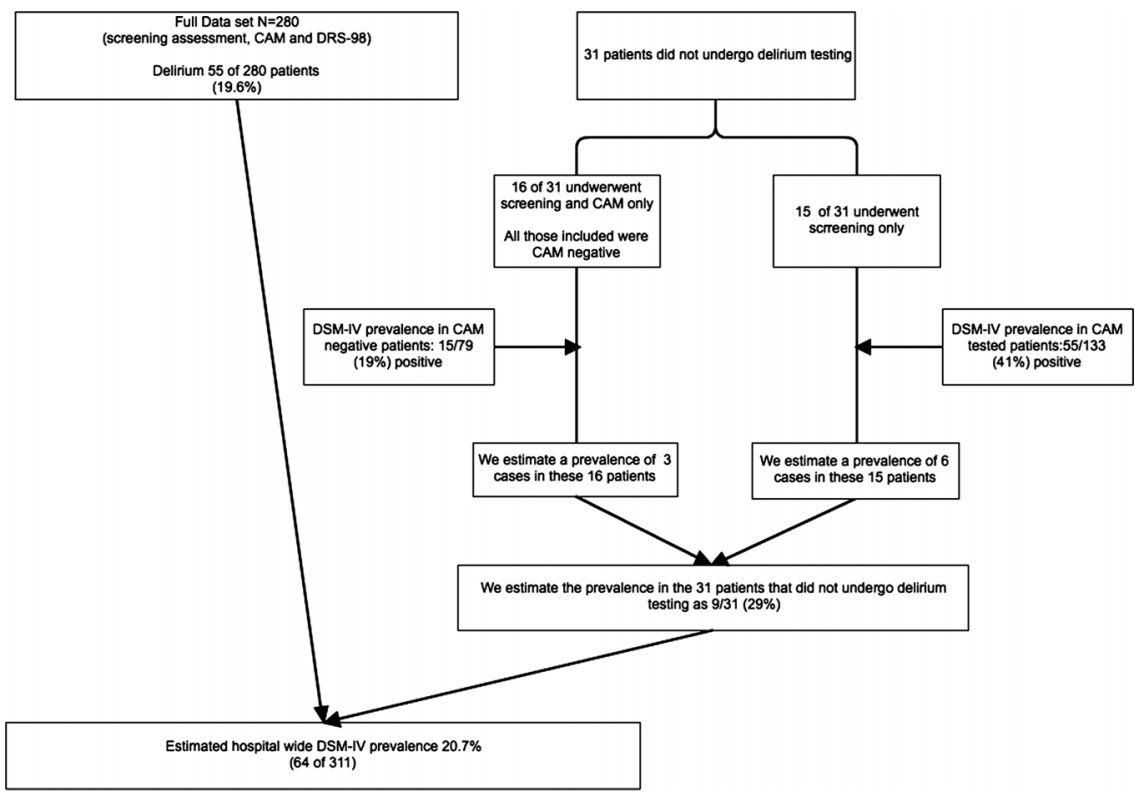




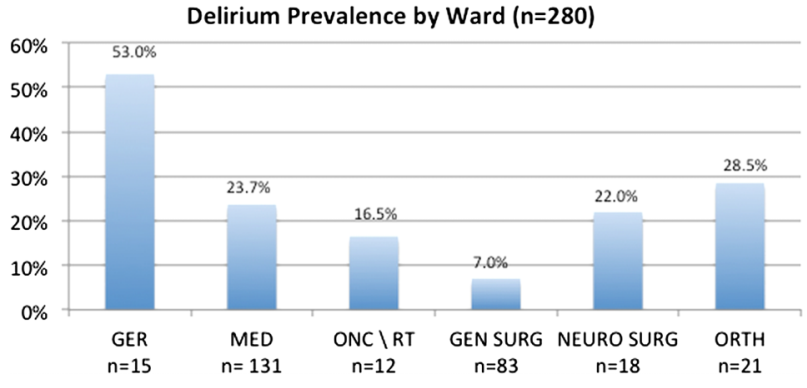

Figure 3 The prevalence of delirium on each unit. GER, geriatric medicine ward $(n=15)$; MED, medical wards $(n=131)$; $\mathrm{ONC/RT}$, oncology and radiotherapy ward $(n=12)$; GEN

SURG, general surgical wards $(n=83)$, including cardiothoracic, vascular surgery and urology wards; NEURO SURG, neurosurgical ward $(n=18)$; ORTH, Orthopaedic ward $(n=21)$ (Total $n=280)$.

older patients in whom cognitive status was definitely known, as explained in the methodology (total $n=203$ ), prior cognitive impairment was an independent predictor of delirium (adjusted OR 15.3; CI 5.2 to 45.4, $\mathrm{p}<0.001$ ) (table 2).

\section{Recognition of delirium}

Of the 55 patients with delirium using DSM-IV criteria, $17(30.9 \%)$ were aware of their cognitive deficit, 35 $(63.6 \%)$ were recognised as confused by nursing staff, and $24(43.6 \%)$ had delirium or one of its synonyms documented in the case notes. Combining these approaches, 43 cases were identified by at least one of these groups (even if the exact term 'delirium' was not used). Logistic regression analyses (table 3) showed that significant independent predictors of delirium documentation in the case notes were severity of inattention and short-term memory impairment and being under medical care. For nurse recognition, independent predictors were the presence of delusions, and the severity of affective lability, inattention and long-term memory impairment. For patient self-recognition, acuity of onset and presence of disorientation were predictors, while sleep-wake cycle disturbance was negatively associated with recognition.
Table 3 Stepwise binary logistic regression of Delirium Rating Scale-Revised-98 item scores and other variables (dementia; medical specialty) for their level of contribution to correct identification of Diagnostic and Statistical Manual of Mental Disorders, Fourth Edition delirium according to research clinician's diagnosis

\begin{tabular}{lrll}
\hline Approach to detection & $\boldsymbol{\beta}$ & $\mathbf{R}^{\mathbf{2}}$ & $\mathbf{p ~ V a l u e}$ \\
\hline Medical case notes & & & \\
DRS-R98 Attention & 0.86 & 0.20 & 0.005 \\
DRS-R98 Short-term memory & 0.56 & 0.24 & 0.04 \\
Medical specialty & 1.70 & 0.27 & 0.006 \\
Constant & -1.14 & & \\
Nurse questioning & & & \\
DRS-R98 Delusions & 0.88 & 0.33 & 0.04 \\
DRS-R98 Affective lability & 1.11 & 0.41 & 0.02 \\
DRS-R98 Attention & 1.08 & 0.44 & 0.01 \\
DRS-R98 Long-term memory & 0.83 & 0.47 & 0.01 \\
Constant & -3.81 & & \\
Patient self-rating & & & \\
DRS-R98 Sleep-wake cycle & -1.31 & 0.19 & 0.01 \\
disturbance & & & \\
DRS-R98 Orientation & 2.02 & 0.25 & $<0.001$ \\
DRS-R98 Onset & 1.32 & 0.30 & $<0.001$ \\
Constant & -4.08 & & \\
\hline On & &
\end{tabular}

Only variables with a significant relationship are shown for each approach to detection. Symptoms are relied upon for delirium detection by different involved parties do not overlap a lot across these methods ( $n=55$ point prevalence cases evaluated).

\section{DISCUSSION}

To our knowledge, this is the first study to assess the point prevalence of delirium in almost an entire hospital rather than extrapolating from selected cohorts or combining data from several studies, and indicates that approximately $20 \%$ of inpatients have delirium at any one time. This prevalence is largely consistent using two different diagnostic tools (CAM, DRS-R98) and gold standard DSM-IV diagnostic criteria, range 18-21\%, indicating that it is a robust figure. In the study, though the CAM performance was lower than DSM-IV or the DRS-R98, it had been administered by rigorously trained geriatricians/senior trainees. The DRS-R98 was independently conducted by psychiatrists with delirium

Table 2 Association using multivariate logistic regression between independent variables and presence of delirium $(n=203$; $\left.\mathrm{R}^{2}=0.52 ; \mathrm{C}_{\mathrm{HL}}=5.19, \mathrm{df}=8, \mathrm{p}=0.738\right)$

\begin{tabular}{llll}
\hline Variable & OR & 95\% Cl & p Value \\
\hline Age & 1.06 & 1.03 to 1.09 & $<0.001$ \\
Speciality & 1 & & \\
Medical/oncology/radiotherapy & $0.004)$ & 0.748 \\
$\quad$ Neurosurgical/orthopaedics & 0.11 & 0.29 to 2.43 & 0.03 to 0.41 \\
$\quad$ Surgical/ophthalmology & 1 & & \\
Dementia & 15.33 & 5.18 to 45.36 & \\
$\quad$ No & &
\end{tabular}


expertise and DRS-R98 training, with ultimate delirium diagnosis determined by independent consensus opinion using DSM-IV criteria. This methodology minimises the short-comings of either diagnostic tool, and the similar overall prevalence of delirium using either tool or DSM-IV criteria (although there was not case-to-case diagnostic concordance) increases the validity of our study outcome. The high level of participation (only five patients refused) supports the generalisability to patients seen in daily clinical practice though the formal methods we employed may not be. A percentage of CAM and DRS-R98 assessments were not conducted in patients who were initially screened, owing to time constraints. An estimation of delirium prevalence among these patients added approximately a further $1 \%$ to the final prevalence figure.

The 'footprint' of delirium in the hospital shows a predilection for older patients especially those with prior cognitive impairment, consistent with the literature that elderly are at the highest risk for delirium, where those over 80 had nearly $35 \%$ prevalence and those under 50 less than $5 \%$. Higher rates were found in medical, neurosurgical and orthopaedic patients, compared to low rates in general surgical patients (corrected OR in surgical versus medical patients is 0.11 (95\% CI 0.03 to $0.41, \mathrm{p}<0.001)$.

Our prevalence figure of $20.7 \%$ represents the burden of delirium in a tertiary referral centre with regional neurosurgery and orthopaedic services and, hence, may not be reflective of all acute hospitals. However, as the prevalence of delirium was similar between general medical patients and these specialised surgical patients, it is possible to extrapolate the results to most hospitals where medical patients are in the majority. The detailed breakdown of the prevalence in various wards/specialities can help predict prevalence patterns in most acute settings. Additionally, as this study used CAM, DRS-R98 and DSM-IV criteria, the results can be directly compared to previous studies from hospital sectors or services that used any of these diagnostic means. Our reported prevalence of $24 \%$ in medical inpatients is similar to that reported in Siddiqi et al 2006 systematic review $^{6}$ and our findings in orthopaedic and neurosurgical patients (28\% and $22 \%$ prevalence, respectively) is also in keeping with previous reports. ${ }^{27-31}$

This study has clear implications for healthcare provision in the acute hospital. First, as expected, there was a linear increase in delirium prevalence with increasing age. As the 'oldest old' segment of the population rapidly expands and hence accounts for a larger proportion of the acute hospital population, hospital delirium rates may increase even further. Our 'footprint' of delirium in the hospital (figure 3) indicates the high-risk wards and patient groups for delirium, and thus the priority areas for staff education and greater access to delirium specialist consultants. Over half of the patients with delirium had pre-existing cognitive impairment (OR 15.3; 95\% CI 5.2 to $45.4, \mathrm{p}<0.001$ ), a known independent risk factor for delirium. ${ }^{32}$ Franco $e t a i^{33}$ found that for each point decrement in Mini Mental State Examination (MMSE) score on admission to the hospital, the DRS-R98 score increased by 0.4 points during the course of the hospitalisation. ${ }^{33}$ However, medical chart review indicated that the responsible medical team did not generally appear to be aware of or have specifically tested for cognitive impairment, consistent with previous reports. ${ }^{34}$ Thus, if we overly focus delirium screening on patients with known cognitive impairment, we may miss cases unless practices for detecting cognitive impairment also improve.

We found $63.6 \%$ of patients with delirium were recognised to be confused or delirious by nursing staff, whereas $43.6 \%$ had confusion documented in the medical case notes. Even if some recognised, but undocumented, delirium cases occurred, it suggests that delirium is not a high diagnostic and therapeutic priority, despite its treatability and relevance to outcomes, especially for poorer prognosis in the elderly. The multidomain assessment within the DRS-R98 provided a more detailed phenomenological understanding as to which symptoms are noticed by whom. Both medical and nursing staff detect delirium by noticing inattentiveness, though nursing staff may rely more heavily on unusual behaviours or communications (lability of affect and delusions), possibly reflecting their more prolonged patient contact and higher social engagement during caring tasks. Prior cognitive impairment is associated with higher rates of nurse detection. This may indicate that nurses recognise the frequency of delirium occurrence in dementia and the overlap between these syndromes, but it may also indicate that the nurses were actually recognising cognitive impairment when they were asked if the patient was 'delirious or acutely confused', without explicitly recognising delirium. Inouye et $a l^{16}$ previously reported that hypoactive, inattentive delirious patients are least likely to be detected by nursing staff. Other researchers feel that nurses over-rely on orientation in assessing delirium. ${ }^{35}$ Medical staff were more likely than nursing staff to detect delirium in the presence of short-term memory impairment and inattention. This may reflect the focus of the medical round: brief, direct questioning of patients, which may unmask attention and cognitive deficits. It may also indicate that doctors regard lability of affect as a separate mood disturbance rather than a delirium feature. Interestingly, patients themselves seemed to equate 'confusion' with disorientation, sleep-wake cycle change and recent deterioration in cognitive function (acuity of onset)they may not be able to describe what happened but they are aware of a major shift (figure 4).

A limitation is that this study was not primarily designed to ascertain staff detection competencies. Nurses were asked, 'Do you think this patient is delirious or acutely confused?' as part of standardised questioning in the screening phase (box 1). This is not a validated tool for determining staff detection of delirium. Our 
Delirium prevalence by age $(n=280)$

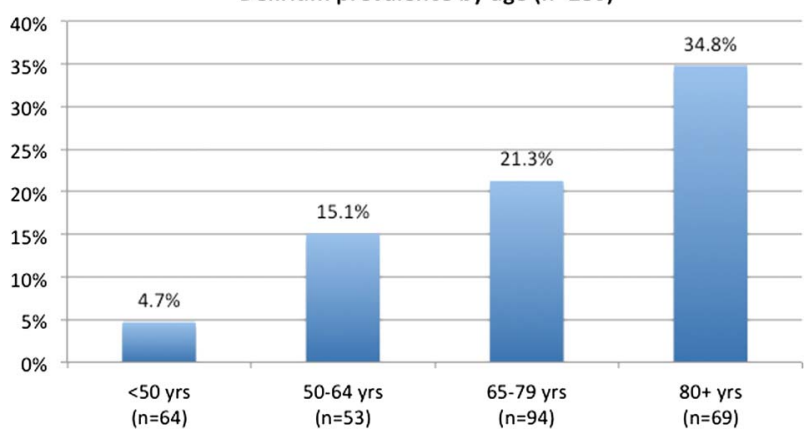

Figure 4 Delirium prevalence stratified by age group.

analysis of medical team recognition relied on documentation in the medical charts, which is a relatively insensitive technique.

Other limitations of this study relate to feasibility to facilitate this ambitious study. We used an initial screening phase so that CAM and DRS-R98/DSM-IV assessments were only performed on patients who had evidence of impaired attention or subjective/objective 'confusion'. Thus, it is possible that some patients with delirium were missed. However, inattention is a cardinal, mandatory feature for delirium, and patients without inattention cannot, by definition, have CAM-defined or DSM-IV-defined delirium, so our screening policy is unlikely to have missed any significant number of cases. Only $86.9 \%$ of inpatients underwent initial screening assessment. Reasons for exclusion, such as stroke-related aphasia or imminent death, suggest that delirium prevalence would be higher in excluded patients. Similarly, patients in the ICU and burns isolation unit were specifically excluded for infection-control purposes. Previous work suggests that delirium prevalence is up to $80 \%$ in ICU patients. ${ }^{36} 37$ Thus, the true point prevalence of delirium was likely to be in excess of $20 \%$ for the whole hospital.

Finally, as well as the $20 \%$ of patients who met DSM-IV criteria for delirium, a further $10 \%$ had some delirium features using the DRS-R98 defined as SSD by score range. Some of these may have been recovering from or about to develop FSD, or indeed may never have reached full diagnostic criteria. Considering that SSD accounted for one-third of all inpatients that exhibited delirium features, further work on the significance of SSD is warranted, namely greater clarification of specific diagnostic criteria, temporal course, phenotype presentation and, particularly, associated mortality risk. Studies show that outcomes in SSD are intermediate between having no delirium and FSD. ${ }^{38}$ This means that we have identified approximately $30 \%$ of the hospital inpatient population who, if undetected and untreated, may incur significant morbidity and mortality.

Thus, delirium is a common problem in hospitalised patients, as demonstrated robustly in this study, regardless of the diagnostic test used. The 'footprint' across the hospital patient population confirms the high-risk patient groups and hence the particular targets for enhanced staff training in delirium prevention, detection and treatment.

\section{Author affiliations}

${ }^{1}$ Centre for Gerontology and Rehabilitation, University College Cork,

St. Finbarr's Hospital, Cork, Ireland

${ }^{2}$ Department of Medical Gerontology, Waterford Regional Hospital, Waterford, Ireland

${ }^{3}$ Department of Geriatric Medicine, Cork University Hospital, Cork, Ireland ${ }^{4}$ Department of Psychiatry, University of Limerick, Limerick, Ireland ${ }^{5}$ Clare Mental Health Services, Ennis Regional Hospital, Clare, Ireland ${ }^{6}$ Department of Psychiatry, University Hospital Limerick, Limerick, Ireland ${ }^{7}$ School of Mathematical Sciences, University College Cork, Cork, Ireland ${ }^{8}$ Department of Neurosciences, Lilly Research Laboratories, Indianapolis, Indiana, USA

${ }^{9}$ Indiana University School of Medicine, Indianapolis, Indiana, USA

Acknowledgements The authors would also like to acknowledge Professor William Molloy for advising on study methodology.

Contributors All the authors substantially contributed to study conception and design, data collection, statistical analysis and interpretation, drafting the initial manuscript and subsequent critical revision of the manuscript and final approval. KO performed statistical analysis, contributed to drafting the original manuscript, performed critical revisions and has approved the final manuscript. PTT had a major contribution to study design and interpretation of data made critical revisions to the manuscript and approved the final version. MO, JM, ST and ML majorly involved in data acquisition, critical revisions and final approval of the manuscript.

Reproducible Research Statement Study protocol: Available from Dr. Niamh O'Regan (see above). Data set: unavailable. Requests for single reprints: Dr. Niamh O'Regan (see above).

Funding This research received no specific grant from any funding agency in the public, commercial or not-for-profit sectors.

Competing interests PTT is a full-time salaried employee and minor shareholder at Eli Lilly and Company. PTT holds the copyright for the Delirium Rating Scale-Revised-98 but does not charge a fee for a not-for-profit use. No other authors have competing interests to disclose.

Ethics approval Clinical Research Ethics Committee of the Cork Teaching Hospitals.

Provenance and peer review Not commissioned; externally peer reviewed.

Data sharing statement There are no additional data available.

\section{REFERENCES}

1. Trzepacz PMD, Leonard MD. Textbook of psychosomatic medicine. 2nd edn. Washington, DC: American Psychiatric Publishing press, 2010.

2. Fong TG, Jones RN, Marcantonio ER, et al. Adverse outcomes after hospitalization and delirium in persons with Alzheimer disease. Ann Intern Med 2012;156:848-56.

3. Gonzalez M, Martinez G, Calderon J, et al. Impact of delirium on short-term mortality in elderly inpatients: a prospective cohort study. Psychosomatics 2009;50:234-8.

4. Kean J, Ryan K. Delirium detection in clinical practice and research: critique of current tools and suggestions for future development. J Psychosom Res 2008;65:255-9.

5. Young J, Murthy L, Westby M, et al. Diagnosis, prevention, and management of delirium: summary of NICE guidance. BMJ 2010;341:c3704.

6. Siddiqi N, House AO, Holmes JD. Occurrence and outcome of delirium in medical in-patients: a systematic literature review. Age Ageing 2006;35:350-64.

7. Wilde N, Strauss E. Functional equivalence of WAIS-III/WMS-III digit and spatial span under forward and backward recall conditions. Clin Neuropsychol 2002;16:322-30.

8. Wiechmann A, Hall JR, O'Bryant SE. The utility of the spatial span in a clinical geriatric population. Neuropsychol Dev Cogn B Aging Neuropsychol Cogn 2011;18:56-63. 
9. Katzman R, Brown T, Fuld $\mathrm{P}$, et al. Validation of a short Orientation-Memory-Concentration Test of cognitive impairment. Am J Psychiatry 1983;140:734-9.

10. Hodkinson HM. Evaluation of a mental test score for assessment of mental impairment in the elderly. Age Ageing 1972;1:233-8.

11. Meagher DJ, Leonard M, Donnelly S, et al. A comparison of neuropsychiatric and cognitive profiles in delirium, dementia, comorbid delirium-dementia and cognitively intact controls. J Neurol Neurosurg Psychiatry 2010;81:876-81.

12. Inouye SK. The confusion assessment (CAM): training manual and coding guide. New Haven: Yale University School of Medicine, 1991

13. Inouye SK, van Dyck $\mathrm{CH}$, Alessi $\mathrm{CA}$, et al. Clarifying confusion: the confusion assessment method. A new method for detection of delirium. Ann Intern Med 1990;113:941-8.

14. Ryan K, Leonard M, Guerin S, et al. Validation of the confusion assessment method in the palliative care setting. Palliat Med 2009;23:40-5.

15. Rolfson DB, McElhaney JE, Jhangri GS, et al. Validity of the confusion assessment method in detecting postoperative delirium in the elderly. Int Psychogeriatr 1999;11:431-8.

16. Inouye SK, Foreman MD, Mion LC, et al. Nurses' recognition of delirium and its symptoms: comparison of nurse and researcher ratings. Arch Intern Med 2001;161:2467-73.

17. Trzepacz PT, Dew MA. Further analyses of the Delirium Rating Scale. Gen Hosp Psychiatry 1995;17:75-9.

18. Trzepacz PT, Mittal D, Torres R, et al. Validation of the Delirium Rating Scale-revised-98: comparison with the delirium rating scale and the cognitive test for delirium. J Neuropsychiatry Clin Neurosci 2001;13:229-42.

19. Adamis D, Treloar A, MacDonald AJ, et al. Concurrent validity of two instruments (the Confusion Assessment Method and the Delirium Rating Scale) in the detection of delirium among older medical inpatients. Age Ageing 2005;34:72-5.

20. Trzepacz PT, Mulsant BH, Dew MA, et al. Is delirium different when it occurs in dementia? A study using the delirium rating scale. J Neuropsychiatr Clin Neurosci 1998;10:199-204.

21. Jorm AF. A short form of the Informant Questionnaire on Cognitive Decline in the Elderly (IQCODE): development and cross-validation. Psychol Med 1994;24:145-53.

22. Jorm AF. The Informant Questionnaire on Cognitive Decline in the Elderly (IQCODE): a review. Int Psychogeriatr 2004;16:275-93.

23. Charlson ME, Pompei P, Ales KL, et al. A new method of classifying prognostic comorbidity in longitudinal studies: development and validation. J Chronic Dis 1987;40:373-83.
24. Hosmer DLS. Applied logistic regression. 2nd edn. New York: John Wiley and Sons, 2000.

25. Nagelkerke N. A note on a general definition of the coefficient of determination. Biometrika 1991;78:691-3.

26. Fry JC. Biological data analysis. A practical approach. Oxford: Oxford University Press, 1999

27. Furlaneto ME, Garcez-Leme LE. Delirium in elderly individuals with hip fracture: causes, incidence, prevalence, and risk factors. Clinics (Sao Paulo) 2006;61:35-40.

28. Gustafson $\mathrm{Y}$, Berggren D, Brannstrom B, et al. Acute confusional states in elderly patients treated for femoral neck fracture. J Am Geriatr Soc 1988;36:525-30.

29. Fisher BW, Flowerdew G. A simple model for predicting postoperative delirium in older patients undergoing elective orthopedic surgery. J Am Geriatr Soc 1995;43:175-8.

30. Kalisvaart KJ, Vreeswijk R, de Jonghe JF, et al. Risk factors and prediction of postoperative delirium in elderly hip-surgery patients: implementation and validation of a medical risk factor model. J Am Geriatr Soc 2006;54:817-22.

31. Marcantonio ER, Flacker JM, Wright RJ, et al. Reducing delirium after hip fracture: a randomized trial. J Am Geriatr Soc 2001;49:516-22.

32. Witlox J, Eurelings LS, de Jonghe JF, et al. Delirium in elderly patients and the risk of postdischarge mortality, institutionalization, and dementia: a meta-analysis. JAMA 2010;304:443-51.

33. Franco JG, Valencia C, Bernal C, et al. Relationship between cognitive status at admission and incident delirium in older medical inpatients. J Neuropsychiatr Clin Neurosci 2010;22:329-37.

34. Sampson EL, Blanchard MR, Jones L, et al. Dementia in the acute hospital: prospective cohort study of prevalence and mortality. $\mathrm{BrJ}$ Psychiatry 2009;195:61-6.

35. Souder E, O'Sullivan PS. Nursing documentation versus standardized assessment of cognitive status in hospitalized medical patients. Appl Nurs Res 2000;13:29-36.

36. Ely EW, Inouye SK, Bernard GR, et al. Delirium in mechanically ventilated patients: validity and reliability of the confusion assessment method for the intensive care unit (CAM-ICU). JAMA 2001;286:2703-10.

37. Pisani MA, Murphy TE, Araujo KL, et al. Benzodiazepine and opioid use and the duration of intensive care unit delirium in an older population. Crit Care Med 2009;37:177-83.

38. Cole M, McCusker J, Dendukuri N, et al. The prognostic significance of subsyndromal delirium in elderly medical inpatients. J Am Geriatr Soc 2003;51:754-60. 